\title{
Commentary: Obesity and cardiac surgery-Double trouble for the surgeon and arrhythmologist
}

\author{
Hussam Ali, MD, FESC, FEHRA, FAIAC, and Riccardo Cappato, MD, FESC, FHRS
}

\author{
From the Arrhythmia \& EP Research Center, IRCCS Humanitas Research \& University Hospital, Milan, Italy. \\ Disclosures: Authors have nothing to disclose with regard to commercial support. \\ Received for publication April 7, 2019; accepted for publication April 8, 2019; available ahead of print May 21, \\ 2019. \\ Address for reprints: Hussam Ali, MD, FESC, FEHRA, FAIAC, Arrhythmia and EP Research Center, IRCCS Hu- \\ manitas Research \& University Hospital, Via A. Manzoni 56, 20089 Rozzano, Milan, Italy (E-mail: hussamali. \\ ep@gmail.com). \\ J Thorac Cardiovasc Surg 2020;159:939-40 \\ $0022-5223 / \$ 36.00$ \\ Copyright (c) 2019 by The American Association for Thoracic Surgery \\ https://doi.org/10.1016/j.jtcvs.2019.04.019
}

Postoperative atrial fibrillation (PoAF) is a common arrhythmia that may complicate the clinical course of patients undergoing cardiac surgery. It has been associated with adverse outcomes including a significant increase in hospitalization stay, readmissions, and mortality. ${ }^{1}$

Obesity is a growing global health problem with negative impact on the cardiovascular system and an increased incidence of atrial fibrillation (AF). The etiology of atrial fibrillation "vulnerability" in obese subjects is still not completely elucidated, but probably multifactorial. Among proposed mechanisms are associated cardiovascular disease, metabolic syndrome, obstructive sleep apnea, increased adipose epicardial tissue, and inflammatory process leading to atrial remodeling and fibrosis. ${ }^{2}$

In this issue of the Journal, Serban and colleagues ${ }^{3}$ analyze the role of obesity in the development of newonset PoAF early after cardiac surgery. As part of prospective observational projects, Serban and colleagues ${ }^{3}$ included 156 patients (67 of whom were obese) who had new-onset PoAF early after surgery for coronary or valvular heart disease. During the first 5 days after the procedure, continuous cardiac rhythm was assessed in all patients and by 2 blinded operators by means of bedside monitors and additional evaluation of available patient records. Obese patients had a higher PoAF burden and more episodes lasting longer than 1 hour than did nonobese patients, with an occurrence peak on the third day.

Although previous data have shown that obesity is a predictor of PoAF, ${ }^{4}$ the study by Serban and colleagues ${ }^{3}$ may be the first to analyze systemically the impact of obesity on the real burden of PoAF by defining episodes in terms of number and duration during the early days after cardiac surgery. This finding is clinically relevant, because a higher atrial fibrillation burden may correlate to the stroke risk in these patients, necessitating anticoagulation therapy. Furthermore, the obesity degree, as estimated by the body mass index, seems to be a determinant factor, because even a small increase in the body mass index of 0.13 units conditions. patients.

\section{References} 8:28-36.

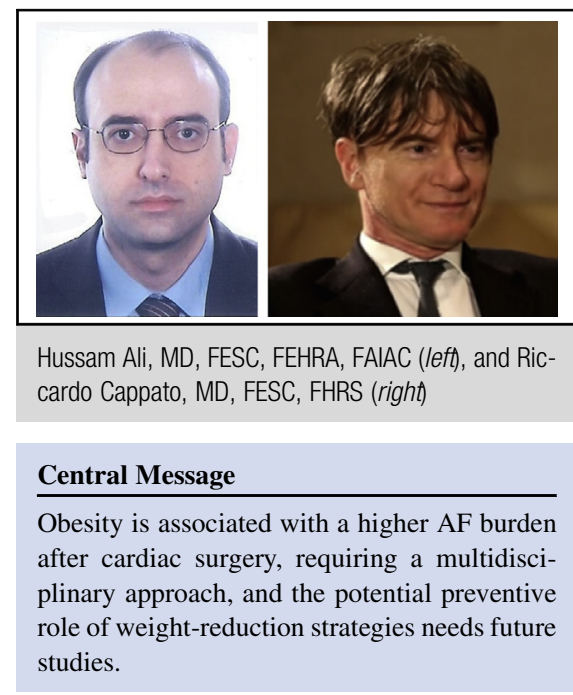

See Article page 930.

$\left(\mathrm{kg} / \mathrm{m}^{2}\right)$ was related to a significant increase in early PoAF burden, indicating a strict correlation between the

Despite the remarkable progress in surgical techniques and pharmacologic treatments, PoAF after cardiac surgery is still a current clinical challenge in terms of prevention and management. As outlined in this study, obese patients are more susceptible to encounter a higher burden and prolonged episodes of PoAF, which may require antiarrhythmic and anticoagulation therapies or early cardioversion. This observation underscores the importance of programming a multidisciplinary approach to manage these high-risk

In spite of the limitations represented by the relatively small sample size of patients and the lack of follow-up data, the study of Serban and colleagues ${ }^{3}$ provides an accurate report of the temporal course and burden of PoAF. Future studies are needed to define the real impact of obesity on PoAF and long-term outcomes, and to determine whether weight reduction strategies before surgery may have a preventive role.

1. Yamashita K, Hu N, Ranjan R, Selzman CH, Dosdall DJ. Clinical risk factors for postoperative atrial fibrillation among patients after cardiac surgery. Thorac Cardiovasc Surg. 2019;67:107-16.

2. Vyas V, Lambiase P. Obesity and atrial fibrillation: epidemiology, pathophysiology and novel therapeutic opportunities. Arrhythm Electrophysiol Rev. 2019; 
3. Serban C, Arinze JT, Starreveld R, Lanters EA, Yaksh A, Kik C, et al. The impact of obesity on early postoperative atrial fibrillation burden. J Thorac Cardiovasc Surg. 2020;159:930-8.e2.
4. Phan K, Khuong JN, Xu J, Kanagaratnam A, Yan TD. Obesity and postoperative atrial fibrillation in patients undergoing cardiac surgery: systematic review and meta-analysis. Int J Cardiol. 2016;217:49-57. 